\title{
RETICULADOS E NÚMEROS BINÁRIOS: UM ATAQUE À CRIPTOGRAFIA RSA
}

\author{
JOSÉ LAUDELINO DE M. NETO
}

\begin{abstract}
Resumo. Descrevemos um ataque ao criptosistema RSA, desenvolvido por May e Ritzenhofen em [1], que consiste em fatorar simultaneamente, utilizando teoria de reticulados, dois números inteiros positivos $N_{1}=p_{1} q_{1}$ e $N_{2}=p_{2} q_{2}$, onde $p_{1}, p_{2}, q_{1}$ e $q_{2}$ são números primos ímpares. Mas, para tal feito é necessário ter duas dicas a respeito destes números $N_{1}$ e $N_{2}$, a saber: $p_{1}$ e $p_{2}$ devem ter alguns bits finais em comum e $q_{1}$ e $q_{2}$ devem ter a mesma quantidade de bits.
\end{abstract}

\section{INTRODUÇÃO}

Nos dias atuais, a criptografia se encontra em voga, pois basta reparar que ao iniciar uma conversa no aplicativo de mensagens WhatsApp, somos surpreendidos com um aviso alertando que "As mensagens e as chamadas são protegidas com a criptografia de ponta a ponta (...)". E o que é criptografia? Podemos dizer que é a arte de codificar uma mensagem, transformando um texto legível e entendível em um texto que fique não compreensível para leitores não autorizados. Apenas leitores autorizados terão acesso ao texto original.

Exemplificando o que foi dito no parágrafo acima: o seguinte texto original "BOM" é cifrado, por algum método criptográfico, no texto " $A B B D L G$ ". Então, somente pessoas autorizadas terão como descriptografar o texto cifrado e recuperar a mensagem original. Os leitores não autorizados, terão acesso apenas ao texto cifrado e não compreensível.

Um dos métodos criptográficos mais famosos é o RSA, criado por Rivest, Shamir e Adleman. Sua segurança consiste na dificuldade em fatorar um número como um produto de primos, pois o cerne do RSA é um número $N$ positivo, produto de dois números primos ímpares, $p$ e $q$ [4]. Este número $N=p q$ é chamado de $R S A$ moduli.

Desde o surgimento de técnicas criptográficas, pesquisam-se maneiras de quebrar a criptografia e desvendar o texto cifrado. Não é diferente com o RSA e é isso que apresentaremos neste texto, um ataque a criptografia RSA, desenvolvida por May e

Data de aceitação: Agosto de 2021.

Palavras chave. Álgebra Linear; Teoria dos Números; Congruência. 
Ritzenhofen [1], na tentativa de fatorar um $R S A$ moduli $N=p q$ utilizando números binários e teoria de reticulados.

\section{Reticulados}

Ressaltamos que, na literatura matemática, o termo reticulado é usado em duas situações distintas. Numa delas, um assunto se refere a conjuntos parcialmente ordenados [5], que não é o do nosso interesse, e na outra se refere a subconjuntos de um espaço vetorial real [3]. Portanto, para entender reticulados no contexto de álgebra linear, é necessário ter conhecimento prévio sobre: conjunto de vetores Linearmente Independentes (LI), espaço vetorial, base de um espaço vetorial, norma de um vetor etc.

Um reticulado inteiro $L$ é um subgrupo discreto e adititivo de $\mathbb{Z}^{n}$. Equivalentemente, sejam $d, n \in \mathbb{N}$ e $\mathbf{b}_{1}, \ldots, \mathbf{b}_{d} \in \mathbb{Z}^{n}$ vetores $L I$, um reticulado inteiro $L$ é o conjunto de todas as combinações lineares inteiras dos vetores $\mathbf{b}_{i}$, ou seja,

$$
L=\left\{a_{1} \mathbf{b}_{1}+\ldots+a_{d} \mathbf{b}_{d} ; a_{i} \in \mathbb{Z}\right\} .
$$

Neste caso, dizemos que o conjunto $\left\{\mathbf{b}_{1}, \ldots, \mathbf{b}_{d}\right\}$ é uma base do reticulado $L$.

O Problema do Menor Vetor, em inglês Shortest Vector Problem, é uma das pesquisas na área [2] e consiste em determinar o vetor de menor comprimento de um reticulado. Ou seja, denotamos $\|\mathbf{v}\|$ como a norma euclidiana do vetor v, e o Problema do Menor Vetor consiste em determinar $\mathbf{0} \neq \mathbf{a} \in L$ tal que $\|\mathbf{a}\| \leq\|\mathbf{v}\|$ para qualquer vetor $\mathbf{v}$ de $L$. Adotamos a norma tradicional euclidiana, porém o problema também pode ser proposto para outras normas usuais. Se a é o menor vetor do reticulado $L$, então escrevemos $\|\mathbf{a}\|=\lambda_{1}(L)$.

Para um caso de dimensão $n$ qualquer, resolver o Problema do Menor Vetor não é uma tarefa fácil. Entretanto, para um reticulado bidimensional $L$, no caso em que $n=2$, é possível utilizar um algoritmo chamado de Redução de Gauss, o qual resolve o Problema do Menor Vetor.

O Algoritmo da Redução de Gauss funciona calculando uma sequência de bases $\{\mathbf{a}, \mathbf{b}\}$ de um reticulado bidimensional $L$, que satisfaz a propriedade

$$
\|\mathbf{a}\| \leq\|\mathbf{a}-\mathbf{b}\|<\|\mathbf{b}\|,
$$

e, ao final de uma quantidade finita de iterações, retorna uma base $\{\mathbf{a}, \mathbf{b}\}$ tal que $\|\mathbf{a}\|=\lambda_{1}(L)$.

\section{Algoritmo da Redução de Gauss}

Entrada: Base de um reticulado bidimensional $L,\{\mathbf{a}, \mathbf{b}\}$.

Saída: Base $\{\mathbf{a}, \mathbf{b}\}$ do reticulado $L$ satisfazendo $\|\mathbf{a}\|=\lambda_{1}(L)$.

(1) Se $\|\mathbf{a}\|>\|\mathbf{b}\|$, então troque a por $\mathbf{b}$ e vá para (2). Caso contrário, vá para (2).

(2) Se $\|\mathbf{a}-\mathbf{b}\|>\|\mathbf{a}+\mathbf{b}\|$, então $\mathbf{b}:=-\mathbf{b}$ e vá para (3). Caso contrário, vá para (3).

(3) Se $\|\mathbf{b}\| \leq\|\mathbf{a}-\mathbf{b}\|$, então pare e retorne $\{\mathbf{a}, \mathbf{b}\}$. Caso contrário, vá para (4).

(4) Se $\|\mathbf{a}\| \leq\|\mathbf{a}-\mathbf{b}\|$, então vá para (6). Caso contrário, vá para (5).

(5) Se $\|\mathbf{a}\|=\|\mathbf{b}\|$, então pare e retorne $\{\mathbf{a}, \mathbf{a}-\mathbf{b}\}$. Caso contrário, vá para (6). 
(6) Determine $\mu \in \mathbb{Z}$ tal que $\|\mathbf{b}-\mu \mathbf{a}\|$ é o menor possível, faça $\mathbf{a}:=\mathbf{b}-\mu \mathbf{a}$, $\mathbf{b}:=\mathbf{a}$. Se $\|\mathbf{a}-\mathbf{b}\|>\|\mathbf{a}+\mathbf{b}\|$, faça $\mathbf{b}:=-\mathbf{b}$. Se $\{\mathbf{a}, \mathbf{b}\}$ satisfizer $\|\mathbf{a}\|,\|\mathbf{b}\|<$ $\|\mathbf{a}-\mathbf{b}\|,\|\mathbf{a}+\mathbf{b}\|$, então pare e retorne $\{\mathbf{a}, \mathbf{b}\}$. Caso contrário, refaça (6).

A etapa (6) é um loop, pois é necessário tornar a repetir o procedimento em (6), até chegarmos ao objetivo. O medo seria o da etapa (6) entrar em um loop infinito, porém isso não ocorre, pois o valor de $\|\mathbf{a}\|$ ou $\|\mathbf{b}\|$ diminui a cada iteração e, como existe uma quantidade finita de vetores de $L$ menores que $\|\mathbf{a}\|+\|\mathbf{b}\|$, então o algoritmo irá finalizar após uma quantidade finita de iterações [2]. Além disso, o problema para determinar $\mu$ na etapa (6) é contornado ao considerarmos a Proposição abaixo, pois garante um intervalo de números inteiros positivos para $\mu$. É justo por isso que $\mu$ pode ser calculado de modo eficiente, pois vamos testando todos os valores de $\mu$ deste intervalo garantido pela Proposição, até encontrarmos um $\mu \in \mathbb{Z}$ tal que $\|\mathbf{b}-\mu \mathbf{a}\|$ é o menor possível.

Proposição: Sejam $\mathbf{a}, \mathbf{b}$ vetores tais que $\|\mathbf{b}\|>\|\mathbf{b}-\mathbf{a}\|$. Então, podemos calcular de modo eficiente um inteiro $\mu$ tal que $\|\mathbf{b}-\mu \mathbf{a}\|$ é o menor possível. Além disso, $1 \leq \mu \leq 2 \frac{\|\mathbf{b}\|}{\|\mathbf{a}\|}$

Demonstração: A demonstração detalhada desta Proposição é encontrada na referência [2].

\section{FAtOrAndo DOIS RSA moduli SIMULTANEAMENTE}

Apresentamos o tema principal deste texto, descrito no Teorema abaixo, que foi a técnica desenvolvida por May e Ritzenhofen [1], a fatoração simultânea de dois $R S A$ moduli, onde duas dicas são dadas. No caso, sejam $N_{1}=p_{1} q_{1}$ e $N_{2}=p_{2} q_{2}$ dois $R S A$ moduli e a primeira dica dada é que $p_{1}$ e $p_{2}$ na sua forma binária possuem alguns dígitos finais em comum, ou seja, alguns bits finais iguais. A segunda dica é que $q_{1}$ e $q_{2}$ tem a mesma quantidade de dígitos na sua escrita em binário, o que equivale a dizer que $q_{1}$ e $q_{2}$ tem a mesma quantidade de bits.

Com o intuito de apresentar a prova do Teorema, lembramos a construção do anel de inteiros módulo $n$, onde $n$ é um número inteiro positivo. Dizemos que dois números inteiros $a$ e $b$ são congruentes módulo $n$ se e somente se $n$ divide $a-b$. Neste caso, escrevemos $a \equiv b(\bmod n)$. A relação $\equiv$ é de equivalência e o conjunto das classes de equivalência é denotado por $\mathbb{Z}_{n}$. As operações de soma e multiplicação induzidas de $\mathbb{Z}$ em $\mathbb{Z}_{n}$ o tornam um anel comutativo. Verifica-se que uma classe de equivalência $\bar{a} \in \mathbb{Z}_{n}$ tem inverso multiplicativo se e somente $\operatorname{se~} \operatorname{mdc}(a, n)=1$.

Teorema: Sejam $N_{1}=p_{1} q_{1}$ e $N_{2}=p_{2} q_{2}$ dois RSA moduli distintos, onde $q_{i}$ tem $\alpha$ bits. Suponhamos que $p_{i}$ possuem $t>2(\alpha+1)$ bits finais em comum. Então, $N_{1} e$ $\mathrm{N}_{2}$ podem ser fatorados simultaneamente.

Demonstração: Como $p_{1}, p_{2}$ possuem $t$ bits finais em comum, temos

$$
p_{1}=2^{t} \widetilde{p_{1}}+p \quad \text { e } \quad p_{2}=2^{t} \widetilde{p_{2}}+p .
$$

Assim, $N_{i}=\left(p+2^{t} \widetilde{p}_{i}\right) q_{i}$, implicando que $p q_{i} \equiv N_{i}\left(\bmod 2^{t}\right), i=1$, 2. Sendo $q_{i}$ primos ímpares, então possuem inversos multiplicativos em $\mathbb{Z}_{2^{t}}$. Logo,

$$
N_{1} q_{1}^{-1} \equiv N_{2} q_{2}^{-1}\left(\bmod 2^{t}\right) \Rightarrow\left(N_{1}^{-1} N_{2}\right) q_{1}-q_{2} \equiv 0\left(\bmod 2^{t}\right) .
$$


O conjunto de soluções

$$
L=\left\{\left(x_{1}, x_{2}\right) \in \mathbb{Z}^{2} ;\left(N_{1}^{-1} N_{2}\right) x_{1}-x_{2} \equiv 0\left(\bmod 2^{t}\right)\right\}
$$

forma um grupo aditivo e discreto de $\mathbb{Z}^{2}$. Isto é, $L$ é um reticulado bidimensional.

Afirmamos que $L$ possui os vetores $\mathbf{b}_{1}=\left(1, N_{1}^{-1} N_{2}\right)$ e $\mathbf{b}_{2}=\left(0,2^{t}\right)$ como base. Com efeito, $\mathbf{b}_{1}, \mathbf{b}_{2} \in L$ e são LI. Por outro lado, seja $\left(x_{1}, x_{2}\right) \in L$. Então, $x_{2}=$ $\left(N_{1}^{-1} N_{2}\right) x_{1}-k 2^{t}$, para algum $k \in \mathbb{Z}$. Assim, $\left(x_{1}, x_{2}\right)=x_{1} \mathbf{b}_{1}-k \mathbf{b}_{2}$.

Pela Equação (1), vemos que $\mathbf{q}=\left(q_{1}, q_{2}\right) \in L$. Entretanto, q ainda está indeterminado. Para deteminar explicitamente quem é q, utilizamos o Algoritmo da Redução de Gauss nos vetores $\mathbf{b}_{1}$ e $\mathbf{b}_{2}$. Após aplicarmos o referido algoritmo, obtemos como retorno que q é o menor vetor de $L$, ou seja, $\|\mathbf{q}\|=\lambda_{1}(L)$ (para maiores detalhes desta passagem, ver [1]). Consequentemente, temos material suficiente para fatorar $N_{1}$ e $N_{2}$ simultaneamente.

O Teorema acima nos garante o algoritmo a seguir:

Entrada: dois RSA moduli $N_{1}$ e $N_{2}$ satisfazendo as hipóteses do Teorema.

Saída: $q_{1}$ e $q_{2}$, onde $N_{1}=p_{1} q_{1}$ e $N_{2}=p_{2} q_{2}$.

(1) Calcule $N_{1}^{-1} N_{2} \in \mathbb{Z}_{2^{t}}$.

(2) Considere $L$ o reticulado gerado por $\mathbf{a}=\left(1, N_{1}^{-1} N_{2}\right)$ e $\mathbf{b}=\left(0,2^{t}\right)$.

(3) Utilize o Algoritmo da Redução de Gauss para determinar o menor vetor $\mathbf{q}=\left(q_{1}, q_{2}\right)$ do reticulado $L$.

Para visualizarmos melhor, consideremos um caso prático. Sejam $N_{1}=p_{1} q_{1}=$ 372581 e $N_{2}=p_{2} q_{2}=493571$ dois $R S A$ moduli tais que $p_{1}$ e $p_{2}$ possuem 12 bits finais em comum e $q_{1}$ e $q_{2}$ possuem 4 bits, ou seja, $N_{1}$ e $N_{2}$ satisfazem as condições do Teorema. Sendo assim, estamos aptos para utilizar o algoritmo apresentado acima. Primeiro, calculamos $N_{1}^{-1} N_{2}$ módulo $2^{12}=4096$,

$$
N_{1}^{-1} N_{2} \equiv 1863(\bmod 4096) \text {. }
$$

Seja $L$ o reticulado gerado pelos vetores $\mathbf{a}=(1,1863)$ e $\mathbf{b}=(0,4096)$. Então, chegamos na etapa de aplicar o Algoritmo da Redução de Gauss. Observamos que os vetores a e b satisfazem as etapas (1) a (4) do Algoritmo da Redução de Gauss e, da etapa (4), vamos para a etapa (6), que, como mencionado antes, funciona como um loop até chegarmos a base procurada do reticulado.

Para o loop da etapa (6), vamos iniciar a primeira iteração com $\mathbf{a}_{0}=\mathbf{a}$ e $\mathbf{b}_{0}=\mathbf{b}$. Utilizando a Proposição, obtemos que

$$
1 \leq \mu \leq 2 \frac{\left\|\mathbf{b}_{0}\right\|}{\left\|\mathbf{a}_{0}\right\|} \approx 4 .
$$

Assim, para $\mu \in\{1,2,3,4\}$, testando caso a caso, concluimos que o menor valor possível para $\left\|\mathbf{b}_{0}-\mu \mathbf{a}_{0}\right\|$ é quando $\mu=2$. Logo,

$$
\mathbf{a}_{1}=\mathbf{b}_{0}-2 \mathbf{a}_{0}=(-2,370), \quad \mathbf{b}_{1}=\mathbf{a}_{0}=(1,1863) .
$$

Como $\left\|\mathbf{b}_{1}\right\|$ não é menor que $\left\|\mathbf{a}_{1}-\mathbf{b}_{1}\right\|$, repetimos o procedimento e, para $\mu \in$ $\{1,2, \ldots, 10\}$, analisando caso a caso, temos que o menor valor possível para $\| \mathbf{b}_{1}-$ $\mu \mathbf{a}_{1} \|$ é quando $\mu=5$. Então,

$$
\mathbf{a}_{2}=\mathbf{b}_{1}-5 \mathbf{a}_{1}=(11,13), \quad \mathbf{b}_{2}=\mathbf{a}_{1}=(-2,370) .
$$


Mais uma vez, como $\left\|\mathbf{b}_{2}\right\|$ não é menor que $\left\|\mathbf{a}_{2}-\mathbf{b}_{2}\right\|$, fazemos o procedimento e, para $\mu \in\{1,2, \ldots, 42\}$, calculando todas as opções disponíveis, temos que o menor valor possível para $\left\|\mathbf{b}_{2}-\mu \mathbf{a}_{2}\right\|$ é quando $\mu=17$. Logo,

$$
\mathbf{a}_{3}=\mathbf{b}_{2}-17 \mathbf{a}_{2}=(-189,149), \quad \mathbf{b}_{3}=\mathbf{a}_{2}=(11,13) .
$$

Neste caso, temos que $\left\|\mathbf{a}_{3}\right\|$ e $\left\|\mathbf{b}_{3}\right\|$ são menores que $\left\|\mathbf{a}_{3}-\mathbf{b}_{3}\right\|$ e $\left\|\mathbf{a}_{3}+\mathbf{b}_{3}\right\|$. Então, temos que $\mathbf{q}=(11,13)$ e $(-189,149)$ é uma base do reticulado $L$ que satisfaz $\|\mathbf{q}\|=\lambda_{1}(L)$. Portanto,

$$
N_{1}=372581=p_{1} q_{1} \quad \text { e } \quad N_{2}=493571=p_{2} q_{2},
$$

onde $q_{1}=11, q_{2}=13$ e, consequentemente, $p_{1}=33871$ e $p_{2}=37967$.

Em binário, temos

$$
\begin{aligned}
& p_{1}=(1000010001001111)_{2}, \\
& p_{2}=(1001010001001111)_{2} \\
& q_{1}=(1011)_{2} \\
& q_{2}=(1101)_{2} .
\end{aligned}
$$

\section{REFERÊNCIAS}

[1] Alexander May \& Maike Ritzenhofen, Implicit Factoring: On Polynomial Time Factoring Given Only an Implicit Hint, In Stanislaw Jarecki and Gene Tsudik, editores, Public Key Cryptography, volume 5443 of Lecture Notes in Computer Science, p. 1-14. Springer, 2009.

[2] Daniele Micciancio \& Shafi Goldwasser, Complexity of lattice problems: a cryptographic perspective, Kluwer Academic Publishers, 2002.

[3] Fernando Daniel Moreira Coelho, O Algoritmo LLL e Aplicações, Dissertação de Mestrado, Faculdade de Ciências e Tecnologia, Universidade de Coimbra, 2007.

$<$ http://www.mat.uc.pt/ $\sim$ jsoares/research/mest_Fernando_Coelho.pdf $>$ Acesso em: $12 / 05 / 2021$.

[4] Manoel Lemos, Criptografia, Números Primos e Algoritmos, IMPA, 2010. $<$ https://impa.br/wp-content/uploads/2017/04/PM_04.pdf> Acesso em: 13/06/2021.

[5] Michell Lucena Dias, Introdução à Teoria dos Reticulados e Reticulados de Subgrupos, Trabalho de Conclusão de Curso, Unidade Acadêmica de Matemática, Universidade Federal de Campina Grande, 2013.

$<$ http://mat.ufcg.edu.br/pgmat2/wp-content/uploads/sites/2/2015/06/TCC-Michell.pdf $>$ Acesso em: 12/05/2021.

Departamento de Ciências Exatas

Centro de Ciências Aplicadas e Educação (CCAE)

Universidade Federal da Paraíba (UFPB)

Rio Tinto, PB

Email address: laudelino@dcx.ufpb.br 